Physics in Medicine and Biology, 2016, 61.19: 7074.)

\title{
Optimising Rigid Motion Compensation for Small Animal Brain PET Imaging
}

\author{
Matthew G. Bickell ${ }^{1}$, Lin Zhou ${ }^{1}$, Andre Z. Kyme ${ }^{2} \ddagger$, Bart De \\ Laat $^{1}$, Roger R. Fulton ${ }^{2,3}$, Johan Nuyts ${ }^{1}$ \\ ${ }^{1}$ KU Leuven - University of Leuven, Department of Imaging and Pathology, Nuclear \\ Medicine \& Molecular Imaging, Medical Imaging Research Center (MIRC), Belgium. \\ ${ }^{2}$ Brain \& Mind Centre and the Faculty of Health Sciences, University of Sydney, \\ Australia. \\ ${ }^{3}$ Department of Nuclear Medicine, Westmead Hospital, Australia. \\ E-mail: matthew.bickell@uzleuven.be
}

\begin{abstract}
Motion compensation (MC) in PET brain imaging of awake small animals is attracting increased attention in preclinical studies since it avoids the confounding effects of anaesthesia and enables behavioural tests during the scan. A popular MC technique is to use multiple external cameras to track the motion of the animal's head, which is assumed to be represented by the motion of a marker attached to its forehead. In this study we have explored several methods to improve the experimental setup and the reconstruction procedures of this method: optimising the cameramarker separation; improving the temporal synchronisation between the motion tracker measurements and the list-mode stream; post-acquisition smoothing and interpolation of the motion data; and list-mode reconstruction with appropriately selected subsets. These techniques have been tested and verified on measurements of a moving resolution phantom and brain scans of an awake rat. The proposed techniques improved the reconstructed spatial resolution of the phantom by $27 \%$ and of the rat brain by $14 \%$. We suggest a set of optimal parameter values to use for awake animal PET studies and discuss the relative significance of each parameter choice.
\end{abstract}

\section{Introduction}

Small animal positron emission tomography (PET) systems have become increasingly important in preclinical imaging since they enable in vivo and longitudinal investigations of animal models. Usually anaesthesia or sedatives are used to eliminate animal movement and stress during the scan. Many reports, however, suggest that anaesthesia can have confounding effects on the kinetics of the tracer or drug under study (Alstrup et al. 2013). To avoid this, researchers have tried to either physically restrain the animal during the scan (e.g. (Hosoi et al. 2005)) or, more commonly, to perform a static scan of an anaesthetised animal after allowing tracer uptake while it was awake (e.g. (Toyama et al. 2004)). However, stress induced by restraint can affect the kinetics of the

$\ddagger$ Currently with the Department of Biomedical Engineering, University of California Davis, U.S.A. 
drug or tracer under study, diminishing the advantage gained by avoiding anaesthesia. Moreover, performing static scans does not allow for the kinetics of the uptake to be estimated and is only possible in a few tracers.

Techniques to allow the animal to be awake and unrestrained during the scan have been investigated by various groups. A novel approach to avoid relative motion between a rat's brain and the PET scanner is the RatCAP (Vaska et al. 2004; Schulz et al. 2011), a miniaturised PET scanner which is surgically attached to the rat's head and which moves rigidly with the brain. While this system is promising it has a lower sensitivity than commercial scanners and may inhibit the natural movement of the rat, possibly inducing stress in the animal.

Motion tracking can be used in conjunction with conventional PET scanners to record the three dimensional motion of the awake animal during the scan, and correct for it offline. This motion compensation (MC) approach avoids the need for anaesthesia and minimises stress since the animal is unrestrained (although the animal is often confined to a small space during the scan). Motion tracking approaches can be either marker-based (Weisenberger et al. 2005; Kyme et al. 2011b), where a small marker is attached to the head of the animal and tracked by external cameras, or markerless, where the facial features of the animal are identified and tracked by external cameras (Kyme et al. 2014). Various marker-based (e.g. (Bloomfield et al. 2003; Fulton et al. 2003; Bühler et al. 2004; Kim et al. 2015)) and markerless (e.g. (Olesen et al. 2013; Noonan et al. 2012)) approaches have been investigated for human studies where MC is also desirable.

Kyme et al. (2011b) successfully applied a marker-based technique to rats using the MicronTracker (ClaroNav Inc., Toronto, Canada), a motion tracking system which is well suited to the application (Kyme et al. 2008). In a MC framework there are several potential sources of error related to both optical motion tracking and how the tracking data are applied for motion correction of the PET data, neither of which are encountered in conventional imaging. This paper builds on previous work by analysing these sources of error and investigating methods to minimise them. The key scientific questions we are trying to answer with respect to MC accuracy are: (i) What is the optimal operating distance for motion tracking? (ii) How important is smoothing and interpolation of the pose data? (iii) What is the best way to relate the discretely sampled pose measurements to what is essentially a continuously sampled PET list-mode data stream? And (iv) when using subsets in the reconstruction, how should these be selected from the motion corrected list-mode data? The paper is structured as follows: section 2 outlines the rationale and principles of the improvements; sections 3 and 4 detail the validation experiments and results, respectively; and sections 5 and 6 contain our discussion and conclusions. 


\section{Methods \& Materials}

\subsection{Data Acquisition and Reconstruction}

All scans were performed using the microPET Focus 220 small animal scanner (Preclinical Solutions, Siemens Healthcare Molecular Imaging, Knoxville, TN, USA). The microPET has an isotropic spatial resolution at the centre of the field-of-view (FOV) of $1.3 \mathrm{~mm}$ full-width at half-maximum (FWHM) (Tai et al. 2005). All data were acquired in 48-bit list-mode format.

Motion tracking was performed using the MicronTracker Sx60 (MT), a stereooptical system which tracks a pre-registered planar marker, an example of which is shown in figure 1(b). The MT uses two simultaneously acquired, but spatially offset, images to estimate the 6 degree-of-freedom (DOF) rigid-body pose (3 components of position and 3 components of orientation) of the marker. The pose measurement rate of the MT is dependent on the processing power of the system used to process the data; for this research a rate of between 20 and $40 \mathrm{~Hz}$ was used.

The PET data were reconstructed using the ordered subsets expectationmaximisation (OSEM) iterative algorithm (Shepp et al. 1982; Hudson et al. 1994). A list-mode based implementation of this algorithm was used (Parra et al. 1998; Reader et al. 1998):

$$
\lambda_{j}^{n+1}=\frac{\lambda_{j}^{n}}{\sum_{i}^{I} s_{i} a_{i j}} \sum_{m}^{M} a_{i_{m} j} \frac{1}{\sum_{k}^{J} a_{i_{m} k} \lambda_{k}^{n}}
$$

where $\lambda_{j}^{n}$ is the value of voxel $j$ of the current reconstruction at iteration $n, J$ is the number of voxels in the image, $M$ is the number of measured list-mode events, $i_{m}$ denotes the detector pair $i$ associated with event $m, I$ is the total number of possible detector pairs, $s_{i}$ is the sensitivity factor for detector pair $i$, and $a_{i j}$ is the system matrix element specifying the contribution of the activity in voxel $j$ to the detection of events in detector pair $i$. This formulation enables event-by-event motion correction of each detected photon pair. The resolution was modelled using the image-based convolution technique with a Gaussian kernel (FWHM $=1.3 \mathrm{~mm}$ ) (Reader et al. 2003). The sensitivity image was calculated as described in Rahmim et al. (2004) by transforming it according to each detected pose, and summing these together as a weighted average. Calculation of this time-averaged sensitivity image accounts for most of the computational burden of the reconstruction. To accelerate this calculation we used only every fifth pose measurement; we have not observed this approach degrading reconstruction quality or introducing artefacts. Since we were only interested in relative changes due to MC, absolute quantification was not necessary and so the effects of attenuation, scatter, and randoms were ignored. 


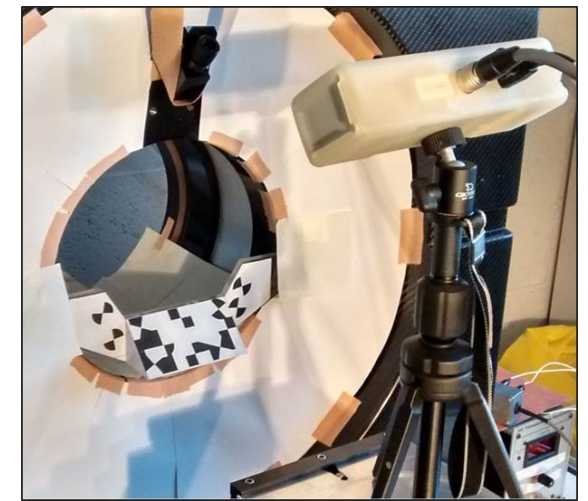

(a)

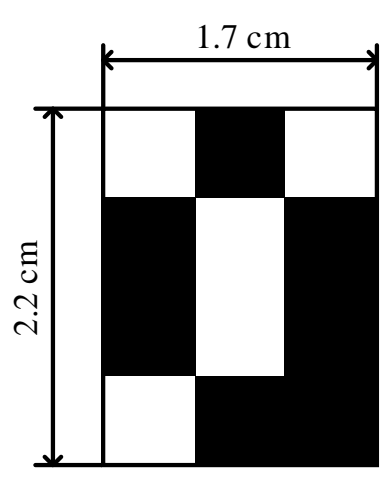

(b)

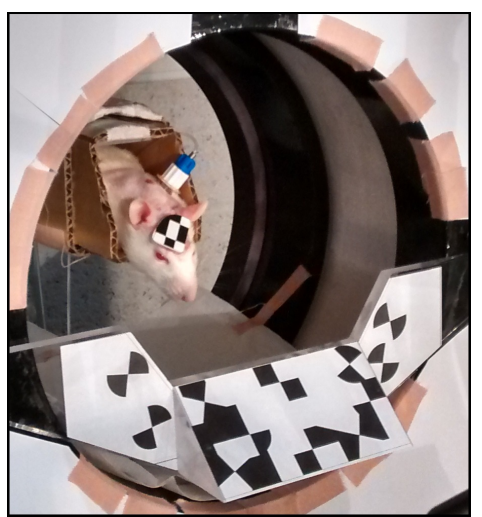

(c)

Figure 1. (a) The MicronTracker in front of the microPET scanner. The large marker attached to the front of the scanner is the reference marker used to aid in transforming from the MT coordinates to the microPET coordinates. (b) The head marker used in rat studies. (c) An unrestrained rat with an attached head marker inside a tube within the microPET. The catheter port can be seen between its shoulders.

\subsection{Spatial calibration}

Pose measurements from motion tracking are reported in the coordinate system of the MT, making it necessary to determine a transformation to convert these data to the microPET coordinate system. For this, a similar procedure to that described by Kyme et al. (2008) and Fulton et al. (2002) was followed. Briefly, a small radioactive microsieve bead was placed at the origin of an MT marker and imaged in the microPET at several discrete locations throughout the FOV. The bead locations in the PET frame were estimated as the centroids of the reconstructed point source images, and the corresponding locations in the tracker frame were measured directly using the MT. A rigid-body transformation matrix relating the two frames was then computed from these paired measurements using the method in (Horn 1987).

To apply the calibration in subsequent experiments in which the relative position of the MT and scanner has changed, we use a reference marker rigidly affixed to the gantry (figure 1(a)). This reference marker can be attached to the scanner highly reproducibly. Measurement of the reference marker pose enables updating of the coordinate calibration according to:

$$
T_{c}^{\prime}=T_{c} T_{R} T_{R}^{\prime-1},
$$

where $T_{R}$ and $T_{R}^{\prime}$ are $4 \times 4$ transformation matrices of the reference marker pose at the time of original calibration and the time of experiment, respectively, using homogeneous coordinates, and $T_{c}$ and $T_{c}^{\prime}$ are the calibration transformation matrices to convert from MT to PET coordinates at the time of original calibration and the time of experiment, respectively. The pose, $\Delta T^{\mathrm{PET}}$, relative to a reference pose in microPET space is given by:

$$
\Delta T^{\mathrm{PET}}=T^{\mathrm{PET}}\left[T_{0}^{\mathrm{PET}}\right]^{-1}=T_{c}^{\prime} T^{\mathrm{MT}}\left[T_{c}^{\prime} T_{0}^{\mathrm{MT}}\right]^{-1}
$$


where the superscript denotes the coordinate space, and $T$ and $T_{0}$ are the transformation matrices of the recorded pose and a chosen reference pose, respectively.

\section{3. $M T$ distance optimisation}

To minimise the stress and discomfort of the rat, the head marker (figure 1(b)) needs to be as small as possible. However, the larger the solid angle subtended by the marker at the MT, the more accurately it can be tracked and the greater the range of motion that can be detected. The MT has a fixed focal length of $700 \mathrm{~mm}$, at which the marker subtends only a small solid angle. To increase the solid angle the MT can be brought closer to the marker, however this will cause out-of-focus blurring. We conducted tests to characterise this trade-off and optimise the MT-marker separation.

An industrial robotic arm (Epson C3-A601S 6-axis, SEIKO Corp., Japan) with repeatability of $\pm 10 \mu \mathrm{m}$ was used to move the marker through several controlled motion sequences and to provide the ground truth of the marker motion. The robotic arm and the MT system were cross-calibrated as described by Kyme et al. (2011b). By repeating the motion sequences, the motion of the marker was measured by the MT at different MT-marker separations, and compared to the applied (robot) motion as a function of the MT-marker separation.

We use $T_{M_{n}}^{\mathrm{MT}}$ and $T_{R_{n}}^{\mathrm{MT}}$ to denote the transformation matrices corresponding to the $n$-th marker pose and the $n$-th robot pose, respectively, in the MT coordinate system (denoted by the superscript). The corresponding incremental motions are denoted by $\Delta T_{M_{n}}^{\mathrm{MT}}$ and $\Delta T_{R_{n}}^{\mathrm{MT}}$, calculated as

$$
\begin{aligned}
\Delta T_{M_{n}}^{\mathrm{MT}} & =T_{M_{n}}^{\mathrm{MT}}\left[T_{M_{n-1}}^{\mathrm{MT}}\right]^{-1} \\
\Delta T_{R_{n}}^{\mathrm{MT}} & =T_{R_{n}}^{\mathrm{MT}}\left[T_{R_{n-1}}^{\mathrm{MT}}\right]^{-1} .
\end{aligned}
$$

Since all calculations occur in the MT coordinate system, below we omit the superscript MT for clarity. To evaluate the discrepancy between measured and applied motion according to (4) and (5), we simulated a uniform spherical grid of points centred on a virtual microPET FOV. We set the radius of the sphere to $10 \mathrm{~mm}$ to approximate the size of the rat brain. Using $X_{p}$ to represent the coordinates of the $p$-th point on the grid, applying the incremental motions to this point results in the transformed points $X_{M_{n p}}$ and $X_{R_{n p}}$ :

$$
\begin{aligned}
& X_{M_{n p}}=\Delta T_{M_{n}} X_{p} \\
& X_{R_{n p}}=\Delta T_{R_{n}} X_{p} .
\end{aligned}
$$

To quantify the similarity of marker and robot poses, we used the offset between these transformed points, calculated by

$$
E_{n p}=\left\|X_{M_{n p}}-X_{R_{n p}}\right\| \text {. }
$$

Since we consider the robot pose to be the gold standard, a smaller $E_{n p}$ indicates improved accuracy of the marker detection. We used the following quantitative measures 
to assess the performance of the marker detection at each MT-marker separation,

$$
\begin{aligned}
& \bar{E}=\frac{1}{N} \sum_{n}^{N} \bar{E}_{n} \\
& \bar{\sigma}=\frac{1}{N} \sum_{n}^{N} \sigma_{n}
\end{aligned}
$$

where $N$ is the total number of incremental poses acquired at that separation, and $\bar{E}_{n}$ and $\sigma_{n}$ are the mean and the standard deviation of $E_{n p}$ over all the points on the grid, respectively.

\subsection{Pose smoothing and interpolation}

To reduce the impact of pose measurement jitter and noise, the measured poses were smoothed in the temporal domain using a finite impulse response pose filter with a Gaussian kernel (Kyme et al. 2011b; Kyme et al. 2011a) (with a FWHM of 100 ms; this kernel is discussed in section 4.4). The smoothing of the rotation matrices is based on the rigid-body pose interpolation approach described Stavdahl et al. (2005) using the "cosine average", but where each rotation matrix is the average of its neighbours, weighted by the kernel.

Poses are typically recorded at a rate of $30 \mathrm{~Hz}$, corresponding to a $33 \mathrm{~ms}$ interval between successive measurements. At this rate it is possible for the subject to move up to $5 \mathrm{~mm}$ between poses (maximum speeds of around $150 \mathrm{~mm} . \mathrm{s}^{-1}$ are observed in awake rat studies). In (Kyme et al. 2011b), each pose measurement was applied in the motion compensation to all list-mode events recorded during the pose interval. However, we hypothesised that interpolating between poses may reduce blur introduced by interpose motion. Cubic splines were used for the interpolation and each DOF was handled independently. While this is not strictly accurate for the rotations (Stavdahl et al. 2005), the angular change from one pose to the next was sufficiently small§ to justify this approximation. In our implementation pose smoothing was always applied before interpolation.

\subsection{Temporal synchronisation}

The poses are recorded by a system which is separate from the microPET controller. Therefore it is necessary to temporally correlate list-mode events and measured poses. This is referred to as the temporal synchronisation between the motion and PET data. As implemented in (Kyme et al. 2011b), to achieve temporal synchronisation a strobe signal was sent from the MT to the gate input of the microPET whenever a pose was recorded; triggering the gate input caused the microPET system to insert a gate tag into the list-mode data stream, which can be used for the data alignment. In

$\S$ The rat motion data from section 3.4 had a maximum angular change of 0.26 radians (mean 0.012 radians, with $75 \%$ of the data below 0.013 radians). This maximum can cause an error of up to 0.024 radians (Stavdahl et al. 2005) in the interpolated angles. 


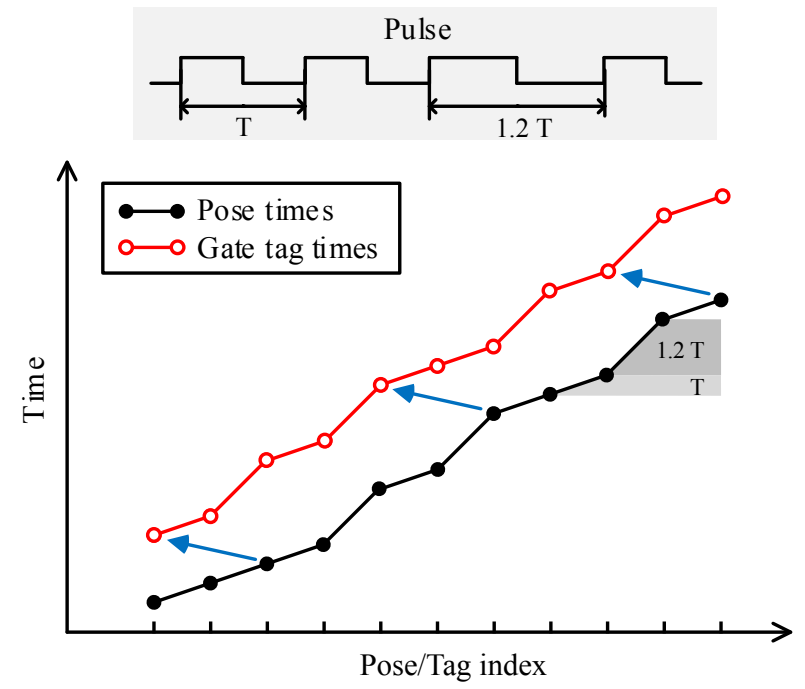

Figure 2. The signal generator produces pulses of a set period (T), but every pulse has a $\frac{1}{32}$ chance of being extended by $20 \%$ (top figure). The extent of the extended intervals has been exaggerated for clarity. Synchronisation between the time stamps of the poses and the gate tags is achieved by aligning the unique pattern of extended pulse lengths present in both streams. In this example there is an offset of two tag indices between the two streams. Such an offset arises if the tracking system starts before the PET acquisition, drops frames, and/or the scanner fails to insert gating tags.

practice, this can be done by aligning the first (or last) pose with the first (or last) gate. However, the reliability of this alignment is difficult to determine since poses or gates may have been missed (typically on the order of 10 gates or poses are missed during scans around an hour long), and only the quality of the reconstruction can suggest that the synchronisation may have been suboptimal.

In order to improve upon this, we developed a signal generator to trigger the MT. The generator produces a block wave pattern in which each block has a probability of $\frac{1}{32}$ of having its length increased by a predetermined factor $(20 \%)$. This is achieved with a 16-bit pseudo-random generator, introducing an irregular and unique interval pattern in the MT poses and, hence, microPET gates, which enables robust alignment (figure 2). The pattern has a period of $2^{16}-1$ triggers, or approximately 35 minutes at a mean triggering frequency of $30 \mathrm{~Hz}$. If a pose or gate was missed, alignment can be maintained based on the trigger pattern before and after the missed element. Since the number of elements in each stream is relatively low (typically on the order of $10^{4}$ ), an exhaustive search can be conducted to find the optimal synchronisation of the two streams, while simultaneously filling in any missing gate tags or poses. The root mean square difference between the absolute times of the gate tags and poses was used to determine the optimal alignment. 


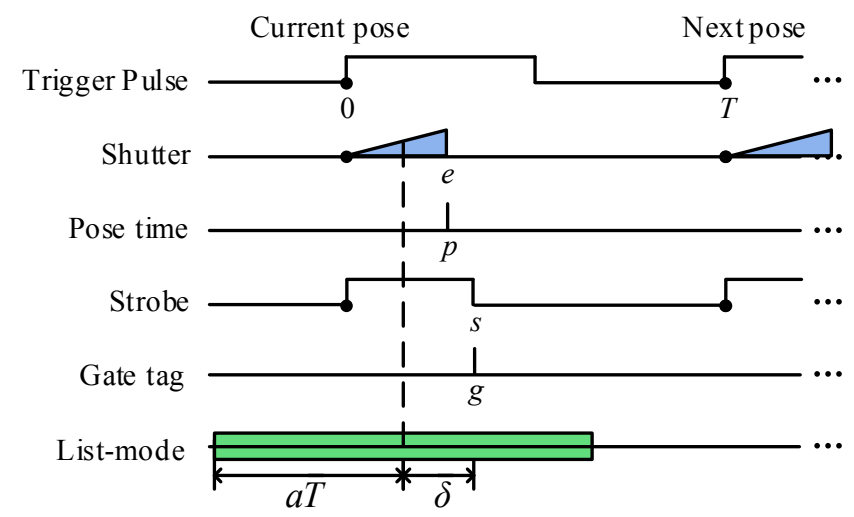

Figure 3. Time sequence of the pose measurement and synchronisation process. Time intervals are shown to scale with the following parameters: $T=30 \mathrm{~ms}, e=8 \mathrm{~ms}$, and $s=10 \mathrm{~ms}$. The last row shows which events should be corrected by the current pose, and the necessary delay $(a T+\delta)$ between the first event and the gate tag. In practice, however, the exact timing of these processes might differ due to unaccounted latencies.

\subsection{Optimised delay time}

Synchronisation latency When triggered by a low-high transition of the signal generator pulses, the MT begins sensor exposure for a duration equal to the specified shutter time ( $e$ in figure 3, typically $4-12 \mathrm{~ms}$ ). After exposure, the recorded image data are time stamped $(p)$ according to the on-board Firewire clock before being transferred to the processing computer for pose computation. At the start of an exposure cycle, the MT also emits a fixed duration strobe pulse ( $s$, typically 10 - $15 \mathrm{~ms}$ ) to trigger the PET system (high-low transition) via the gating input. Ideally, the insertion of the gate tag $(g)$ in response to this trigger is instantaneous. This idealised time sequence of events is illustrated in figure 3. In practice, there may be timing fluctuations from intermediate processes which result in deviations from the ideal scenario. We model the real delay between the inserted gate tags and the pose time stamps as a constant latency $\delta$ (as shown in figure 3), and fit it empirically.

Synchronisation implementation A measured or interpolated pose is applied to the listmode events spanning the interval associated with that pose. With no interpolation this interval is around $30 \mathrm{~ms}$, with interpolation it is usually $1 \mathrm{~ms}$. Therefore a particular pose can be regarded as the average of the motion over the pose interval, starting half an interval before the pose time stamp and ending half an interval after. The first list-mode event to which the pose should be applied is then at half an interval before the pose time stamp. We consider this to be an interval-dependent contribution to the constant latency described above, viz.:

$$
\Delta=-\frac{1}{2} T+\delta
$$

where $T$ is the interval between subsequent poses. 


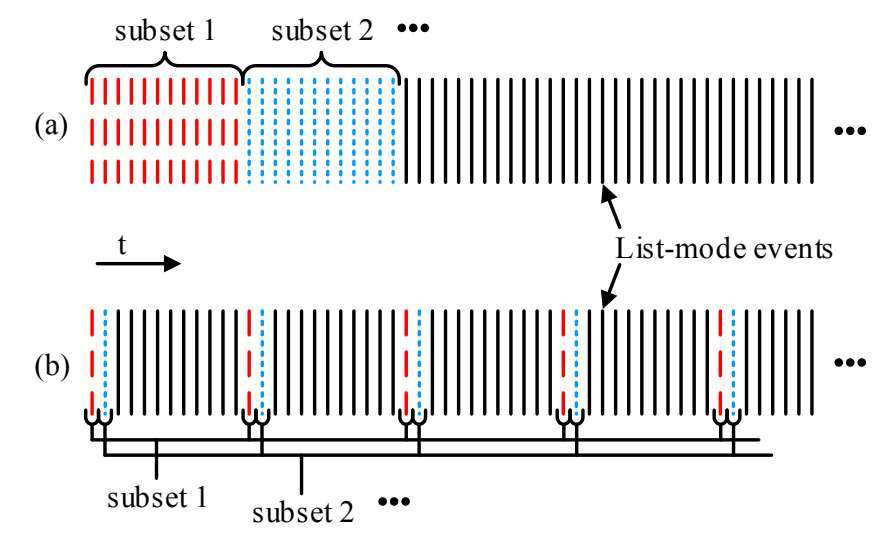

Figure 4. Subset selection. Subsets can be selected as chronological segments (a) or by selecting events spread evenly throughout the list-mode stream (b). The second method ensures that all subsets are subject to a similar range of motion.

\subsection{Subset selection}

The convergence of the reconstruction can be accelerated by using subsets of the listmode data in each iteration (Reader et al. 1998). One possible option is to choose subsets as chronological segments of the list-mode data (figure 4(a)). However, since the average sensitivity image is calculated as the time-weighted average of the sensitivity image transformed by each pose throughout the scan, it is possible to introduce an error if the motion in a given subset differs significantly from the motion present in the average sensitivity image. Therefore, we propose to select each subset from the entire list-mode data set such that it incorporates the motion throughout the scan, as illustrated in figure 4(b). This approach was used in all reconstructions.

\section{Experiments}

\subsection{MT distance optimisation}

To optimise the MT-marker separation we used the Epson robot to manipulate a rat head marker according to known motion while it was synchronously tracked by the MT. Three motion sequences were executed by the robot: (i) arbitrary continuous motion, (ii) measured rat motion, and (iii) step-wise motion in which each pose was held for $5.5 \mathrm{~s}$. All sequences were 2 minutes and involved 6 DOF changes in marker pose. Each motion sequence was repeated multiple times at a different MT-marker separation ranging from $285 \mathrm{~mm}$ to $575 \mathrm{~mm}$. In all cases tracking was performed at $30 \mathrm{~Hz}$. The MT and robot data were post-processed and compared as described in section 2.3.

\subsection{Optimised delay time}

The optimal delay time was determined based on motion compensated reconstructions of a moving point source. A $2 \mathrm{~mm}$ diameter porous bead containing $0.74 \mathrm{MBq}{ }^{18} \mathrm{~F}-\mathrm{FDG}$ was placed at the centre of a large MT marker (diameter $57 \mathrm{~mm}$ ) and scanned within 
the microPET FOV for 2 minutes while undergoing a robot-controlled arbitrary motion in conjunction with motion tracking (strobe duration $15 \mathrm{~ms}$, shutter $8 \mathrm{~ms}$ ). The scan was repeated thirty times with the pose measurement rate varying between $18-39 \mathrm{~Hz}$. Images were reconstructed using the method described in section 2.1 (using 2 subsets and 7 iterations). Reconstructions of each data set were performed using a range of delays between the measured poses and the gate tags. The optimal delay for each data set was determined by finding the reconstruction with the highest maximum value. This is based on the assumption that any motion blur due to suboptimal delays will lower the maximum value of the point source reconstruction.

\subsection{Phantom studies}

In order to quantify the impact of our optimisation strategies on spatial resolution, we performed motion compensated imaging for a hot rod phantom (radius $40 \mathrm{~mm}$, rod diameter ranging from $1.5-3.0 \mathrm{~mm}$ ) moved by a robotic arm in the microPET. The phantom was filled with $48 \mathrm{MBq}$ of ${ }^{18} \mathrm{~F}$-FDG and several 2-minute scans were performed using the same robot-controlled arbitrary motion sequence (maximum offset $40 \mathrm{~mm}$ radially and $14 \mathrm{~mm}$ axially of the phantom marker from the FOV centre) executed at increasing speed. The speed of the phantom was determined post-acquisition and we show results from a slow (mean speed $45.0 \mathrm{~mm} . \mathrm{s}^{-1}$, maximum speed $195.3 \mathrm{~mm} . \mathrm{s}^{-1}$ ) and a fast (mean speed $80.1 \mathrm{~mm} . \mathrm{s}^{-1}$, maximum speed $379.3 \mathrm{~mm} . \mathrm{s}^{-1}$ ) case. For each speed, motion tracking was performed at $28.5 \mathrm{~Hz}$ for three different MT-marker separations: $400 \mathrm{~mm}, 500 \mathrm{~mm}$, and $600 \mathrm{~mm}$. Reconstructions were performed as described in section 2.1 (using 10 subsets and 10 iterations) with a pixel size of [0.4745, 0.4745, 0.796] mm.

\subsection{Rat studies}

All animal experiments were approved by the ethical committee of KU Leuven and performed in accordance with the European Communities Council Directive (86/609/EEC).

A female Wistar rat (weight $275 \mathrm{~g}$ ) was trained over three 30-minute sessions to remain unrestrained in a tube with its head extending into the microPET FOV (Bickell et al. 2015). Since the body temperature of a rat can be used as an indicator of its stress level, Fulton et al. (2009) used peritoneally implanted temperature sensors in rats to show that their body temperature was not elevated to levels indicative of stress while they remained in the tube for awake scanning procedures lasting up to $60 \mathrm{~min}$. The marker shown in figure 1(b) was attached to the rat's shaved forehead using "superglue", which bonds and dries rapidly and usually remains attached for 2 - 3 days. Firm marker attachment is crucial since rats do sometimes try to remove it during the scan. For imaging, the rat was injected with $30 \mathrm{MBq}{ }^{18} \mathrm{~F}-\mathrm{FDG}$ via a surgically implanted jugular catheter, with an access port protruding dorsally from between the scapulae, and scanned for 10 minutes, 65 minutes post injection. During the scan, the marker on the head was tracked using the MT (figure 1(c)). The MT-marker separation was 

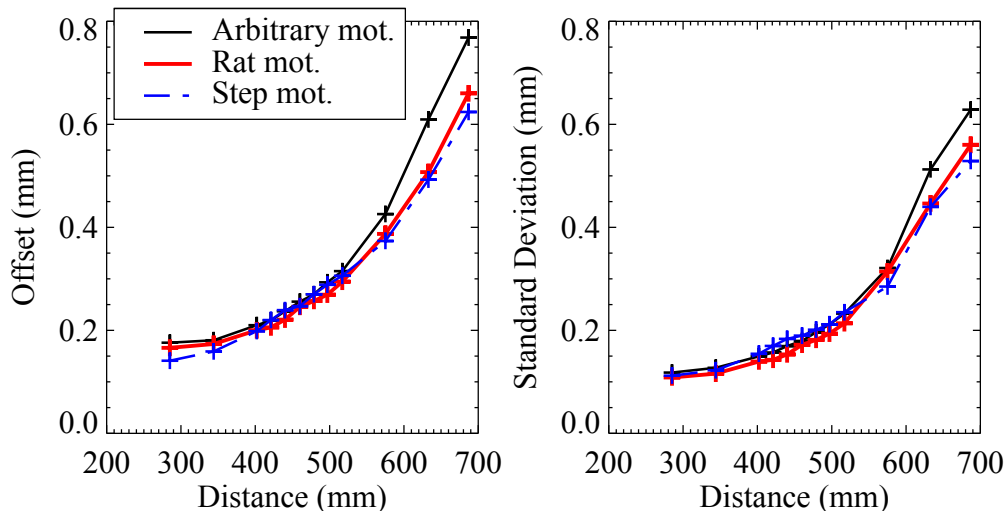

Figure 5. The averaged offsets ( $\bar{E}$, left) and the averaged standard deviations of the offsets $(\bar{\sigma}$, right) for points in the test grid.

$400 \mathrm{~mm}$ and the pose measurement rate was $30 \mathrm{~Hz}$. After scanning the awake animal, the rat was anaesthetised (2.5\% isofluorane in $\mathrm{O}_{2}$ at $2 \mathrm{~L} / \mathrm{min}$ ) and imaged asleep for 10 minutes (starting 89 minutes post injection). Reconstructions were performed as described in section 2.1 (using 10 subsets and 10 iterations) with a pixel size of [0.4745, $0.4745,0.796] \mathrm{mm}$.

\subsection{Pose smoothing and interpolation}

Data from the moving phantom experiments were used to determine the optimal FWHM of the smoothing kernel to be used on the pose data, and to quantify the effect of pose interpolation on the reconstructed spatial resolution. Both the slow and the fast motion cases were investigated. The MT-marker separation used was $400 \mathrm{~mm}$. The motion data were smoothed using a Gaussian kernel with a temporal FWHM varying from $0-120 \mathrm{~ms}$.

With interpolation the measured frequency of $28.5 \mathrm{~Hz}$ was interpolated to $1000 \mathrm{~Hz}$, which ensures that the inter-pose motion never exceeds $0.4 \mathrm{~mm}$ for our phantom scans and $0.15 \mathrm{~mm}$ for a typical rat scan, which is sufficiently small for our purposes.

\section{Results}

\subsection{MT distance optimisation}

For the three motion sequences studied, the pose error $(\bar{E})$ and standard deviation $(\bar{\sigma})$ both reduced as the MT-marker separation reduced despite an increase in out-of-focus blurring (figure 5). Although accuracy improved down to $300 \mathrm{~mm}$ separation, in order to retain the gantry reference marker in the MT FOV the smallest practical MT-marker separation was $400 \mathrm{~mm}$. 


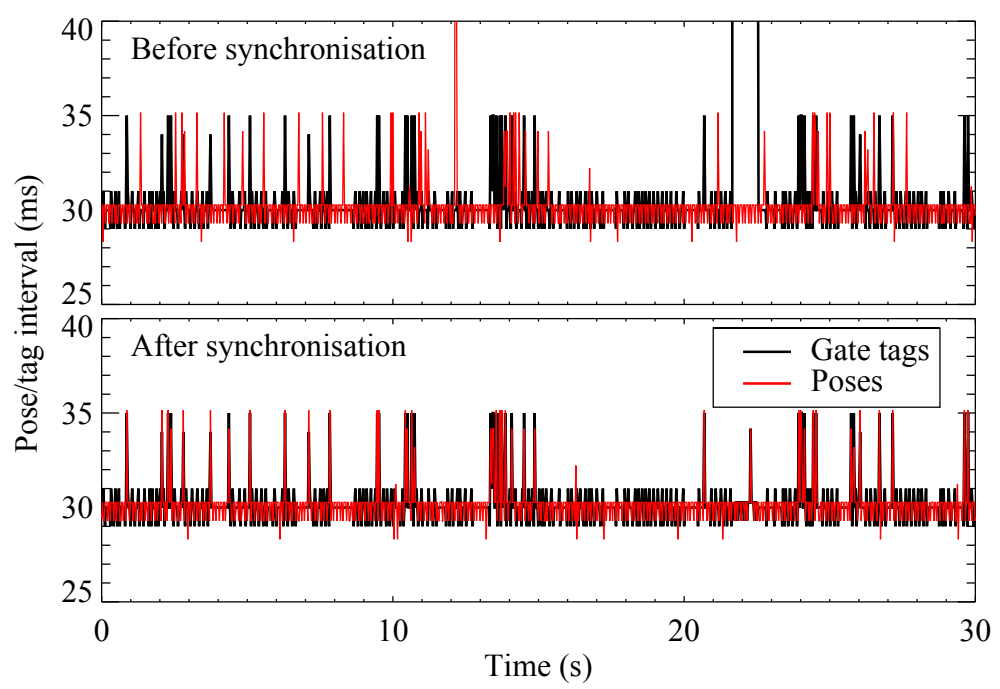

Figure 6. Time intervals between the gate tags and the pose measurements, before (top) and after (bottom) synchronisation. The intermittent increases from $29 \mathrm{~ms}$ to $35 \mathrm{~ms}$ are due to the randomisations in the signal generator. Before synchronisation there was one gap in both the pose stream and the gate tag stream, manifest by the large times at around $12 \mathrm{sec}$ and $22 \mathrm{sec}$, respectively. This was corrected for with synchronisation (bottom).

Table 1. Linear fit parameters for the optimal delay time.

\begin{tabular}{rccc}
\hline & $a$ & $\delta(\mathrm{ms})$ & $r^{2}$ \\
\hline Without interp. & -0.952 & 21.525 & 0.948 \\
With interp. & -0.429 & 23.741 & 0.652 \\
\hline
\end{tabular}

\subsection{Temporal synchronisation}

Based on several long scans we determined that the clocks governing the microPET and MT timestamps differed by a constant scale factor of 1.000181191, with the microPET clock running slightly slower than the MT clock. While this scale may seem small, over 10 minutes it introduces a temporal shift of several pose intervals between the poses and gate tags. We corrected for the clock scale difference before synchronising the data streams. Figure 6 shows an example data stream of gate tags and poses before and after synchronisation. Before synchronisation, both the pose and gate tag data streams contained a gap corresponding to missing poses or gate tags, respectively. By comparing the randomised interval pattern (section 2.5) before and after the gap, the two streams could be synchronised. Any data corresponding to initial gaps were ignored during MC.

\subsection{Optimised delay time}

Figure 7 shows the results of the optimal delay investigation with and without pose interpolation. A linear fit was performed using $\Delta=a T_{0}+\delta$ for each case, where $T_{0}$ is the measured pose interval. The results are shown in table 1 . Clearly the model 


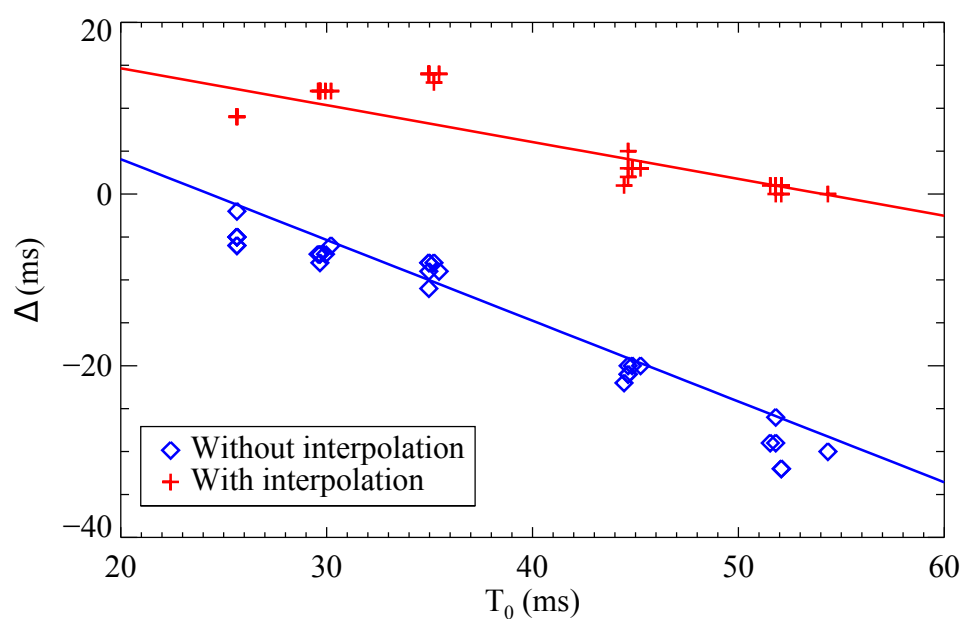

Figure 7. The optimal delay time for thirty point source scans at different measured pose intervals, $T_{0}$, with (red) and without (blue) pose interpolation. The solid lines show the results of the linear fit.

described by (11) does not describe the data well, and the linear fit in table 1 has a poor correlation with the data in the interpolation case. Further investigation is needed to determine an accurate model for this data. For this work, the mean optimal delay values determined for the point source scans at each pose measurement rate were used in phantom and rat studies, i.e. $-9 \mathrm{~ms}$ with no pose interpolation, and $14 \mathrm{~ms}$ with interpolation.

\subsection{Phantom studies}

Figures 8 and 9 show reconstructed images and profiles of the phantom for a slow and fast motion, respectively, and in each case using various combinations of the optimised parameters: the MT-marker separation, pose smoothing, pose interpolation, and the delay applied to the pose time stamps. The images represent a sum of 26 slices $(20.7 \mathrm{~mm})$ along the rods to reduce the impact of noise. By analysing the reconstructions with various pose smoothing kernels and comparing their resolution recovery, a kernel with FWHM of $100 \mathrm{~ms}$ was determined to be optimal for both the slow and the fast motion cases. We also verified that the optimal delay predicted from (11) produced the highest resolution recovery by performing reconstructions with various delays.

Pose smoothing (figures 8/9, row 1 ) resulted in the most dramatic improvement in image quality out of all the optimisation techniques. There was moderate improvement by reducing the MT-marker separation (figures $8 / 9$, row 2 ) and using pose interpolation and an optimised synchronisation delay (figures $8 / 9$, row 3 ). The reconstructed images obtained using full optimisation most closely resembled those of the static phantom.

To quantify the residual motion blur present in the $\mathrm{MC}$ reconstructions, and thus the resolution improvement gained when using the various optimisation techniques, pairs of images were compared by determining the FWHM of the 3D Gaussian kernel needed to smooth one image (the reference) to achieve the lowest pixel-by-pixel root mean 


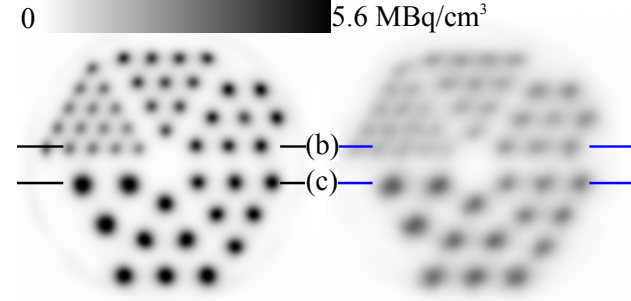

(i) Motion-free

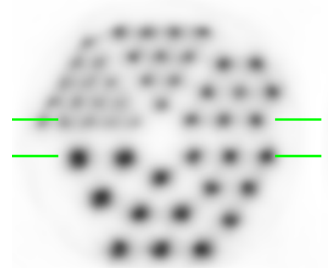

(iii) D: $600 \quad \mathrm{I}: \times$ $\mathrm{S}: \checkmark \quad \Delta: \times$

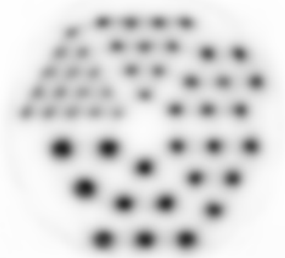

D: $400 \quad \mathrm{I}: \times$ (ii) D: $400 \quad \mathrm{I}: \times$ $\mathrm{S}: \times \Delta: \times$

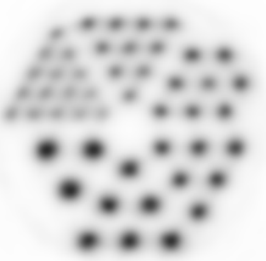

D: $500 \quad \mathrm{I}: \times$

$\mathrm{S}: \checkmark \quad \Delta: \times$

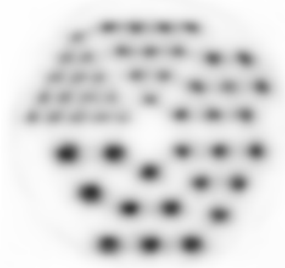

D: $400 \quad \mathrm{I}: \checkmark$

$\mathrm{S}: \checkmark \quad \Delta: \times$

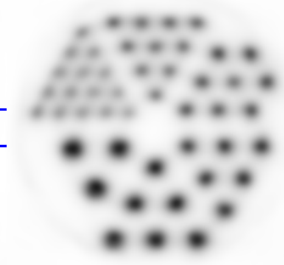

D: $400 \quad$ I: $\times$

$\mathrm{S}: \checkmark \quad \Delta: \times$

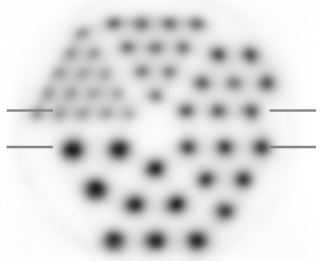

(iv) D: $400 \quad \mathrm{I}: \times$ S: $\checkmark \quad \Delta: \times$

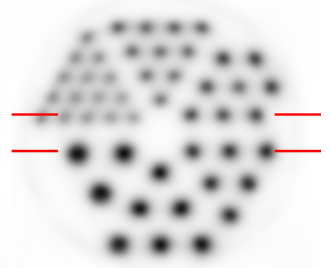

(v) D: $400 \quad \mathrm{I}: \checkmark$

(a)

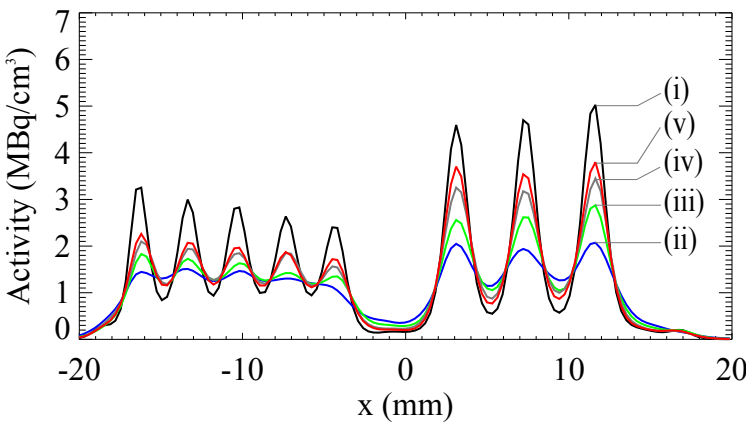

(b)

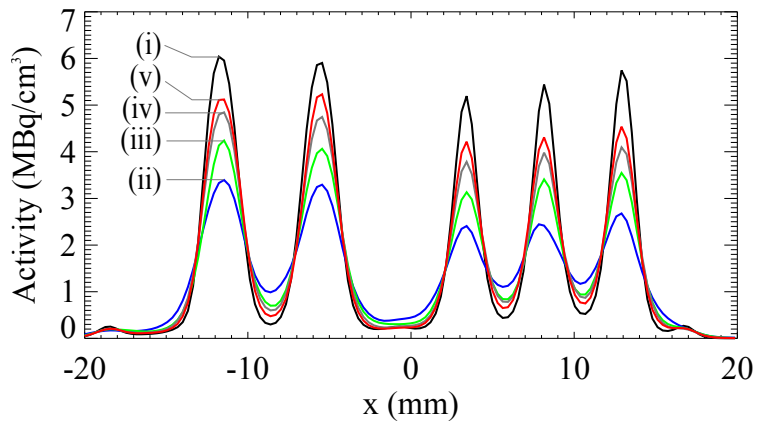

(c)

Figure 8. (a) Reconstructed images of the phantom undergoing slow motion, after summing in the slice direction. The images are shown to the same scale and have been corrected for decay. The applied parameters are shown below each image: D is the MT-marker separation in $\mathrm{mm}$; I refers to pose interpolation; S refers to pose smoothing; and $\Delta$ refers to the optimal delay time. A check indicates that the factor was applied. The coloured lines indicate the location of the profiles through the (b) small and (c) big rods, and the Roman numerals indicate the corresponding profiles in (b) and (c). 


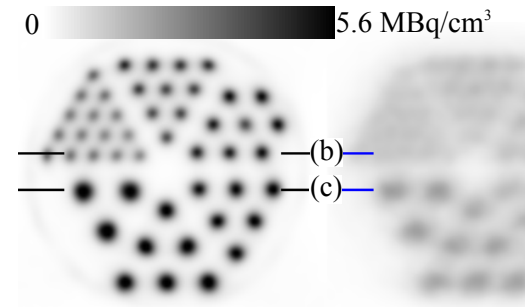

(i) Motion-free

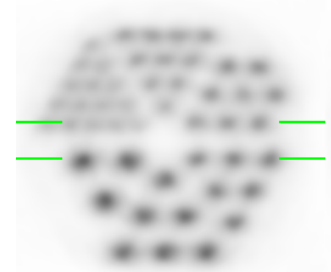

(iii) D: $600 \quad \mathrm{I}: \times$
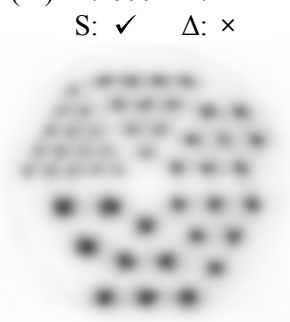

D: $400 \quad \mathrm{I}: \times$ (ii) D: $400 \quad \mathrm{I}: \times$

$\mathrm{S}: \times \Delta: \times$

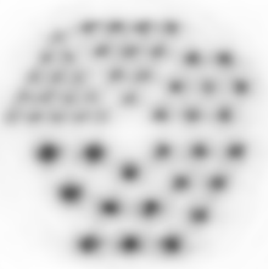

D: $500 \quad \mathrm{I}: \times$

S: $\checkmark \quad \Delta: \times$

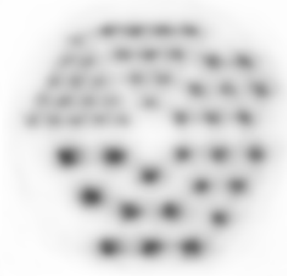

D: 400 I: $\checkmark$

S: $\checkmark \quad \Delta: \times$

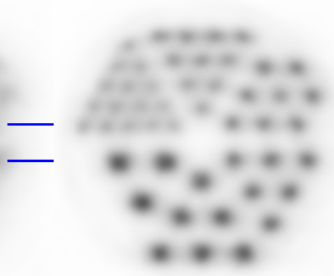

D: $400 \quad \mathrm{I}: \times$

S: $\checkmark \quad \Delta: \times$

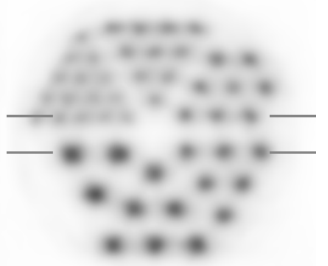

(iv) D: $400 \quad \mathrm{I}: \times$ S: $\checkmark \quad \Delta: \times$

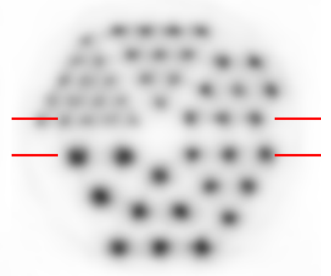

(v) D: 400 I: $\checkmark$

(a)

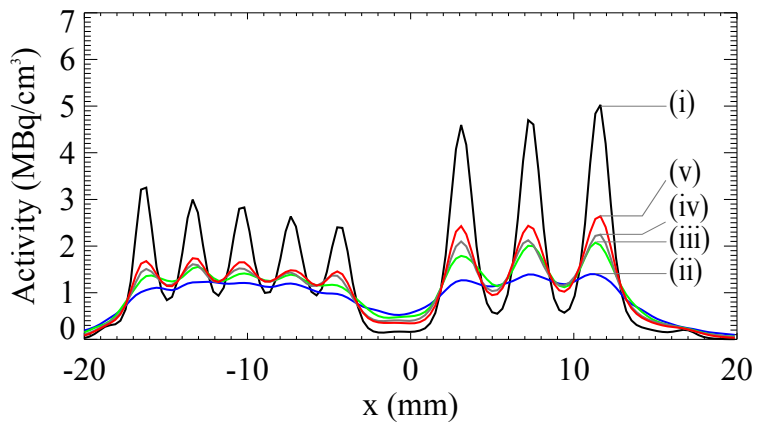

(b)

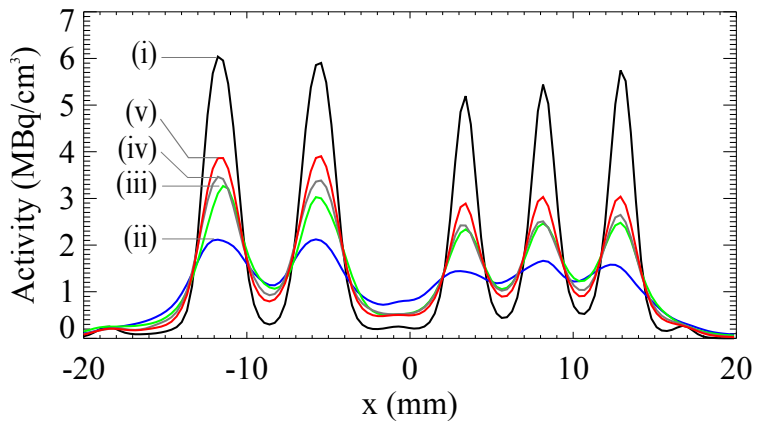

(c)

Figure 9. (a) Reconstructed images of the phantom undergoing fast motion, after summing in the slice direction. The images are shown to the same scale and have been corrected for decay. The applied parameters are shown below each image: D is the MT-marker separation in $\mathrm{mm}$; I refers to pose interpolation; $\mathrm{S}$ refers to pose smoothing; and $\Delta$ refers to the optimal delay time. A check indicates that the factor was applied. The coloured lines indicate the location of the profiles through the (b) small and (c) big rods, and the Roman numerals indicate the corresponding profiles in (b) and (c). 
Table 2. Comparison of resolution recoveries.

\begin{tabular}{|c|c|c|c|c|}
\hline \multirow{2}{*}{\multicolumn{2}{|c|}{ Reference }} & \multirow[b]{2}{*}{ Test image } & \multicolumn{2}{|c|}{ FWHM $(\mathrm{mm})$} \\
\hline & & & Slow & Fast \\
\hline (i) & Digitised & Motion-free & \multicolumn{2}{|c|}{1.5} \\
\hline (ii) & Digitised & Opt. MC & 1.9 & 2.3 \\
\hline (iii) & ) Digitised & Subopt. MC & 2.6 & 3.2 \\
\hline (iv) & ) Opt. MC & Subopt. MC & 1.5 & 1.8 \\
\hline
\end{tabular}

square difference with the other image (the test image). We use a uniform Gaussian kernel since much of the residual blur is due to noise in the pose measurements, and therefore should introduce approximately uniform blurring. A digitised 3D version of the hot rod phantom was used as the reference image and compared to the motion-free, optimal MC (i.e. obtained using all optimisations), and suboptimal MC (i.e. obtained using no optimisations and an MT-marker separation of $400 \mathrm{~mm}$ ) reconstructions. Additionally, the optimal MC reconstructions were used as the reference and compared to the suboptimal MC reconstructions. The results of this analysis are shown in table 2. Comparing (ii) and (iii) in table 2, the spatial resolution improved by approximately $27 \%$, and the magnitude of the improvement was almost identical in the slow and fast motion cases.

\subsection{Rat studies}

Figure 10 shows the reconstructed rat images, together with the recorded head motion and two profiles through the reconstructions. The mean speed of the rat's head during the "awake" scan was $6.79 \mathrm{~mm} . \mathrm{s}^{-1}$ (maximum $136.7 \mathrm{~mm} . \mathrm{s}^{-1}$ ), with a maximum offset of $37 \mathrm{~mm}$ radially and $40 \mathrm{~mm}$ axially of the marker from the FOV centre. The motion-free reconstruction is shown together with the $\mathrm{MC}$ reconstruction using no optimisations and all optimisations. While the motion-free and $\mathrm{MC}$ reconstructions correlate closely to each other, some small differences are observed. These are largely due to residual errors in the MC, but, since the scans were separated by 14 minutes, slight changes in the FDG distribution may have also contributed to these differences. Using the same quantification approach described in section 4.4, with the motion-free reconstruction as the reference image, the improvement in spatial resolution using $\mathrm{MC}$ with optimisation, compared to no optimisation, was $14 \%$ (from a kernel with FWHM of $1.08 \mathrm{~mm}$ to $0.93 \mathrm{~mm})$.

\section{Discussion}

In this study we have presented various optimisation techniques to improve MC reconstructions for PET imaging. Hardware (MT-marker separation and temporal synchronisation) and software (latency estimation between the poses and gate tags, pose smoothing and interpolation, and subset selection during reconstruction) optimisations 


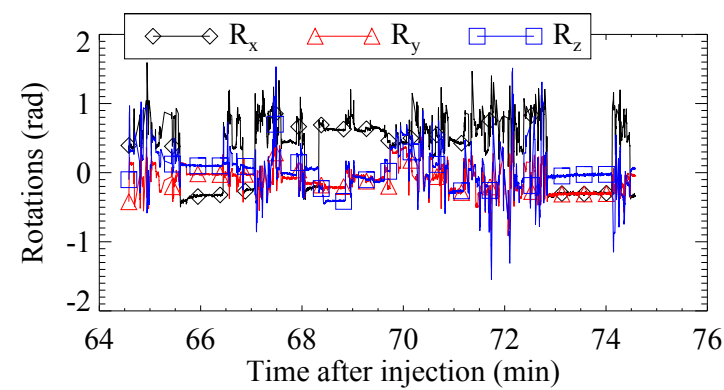

(a)

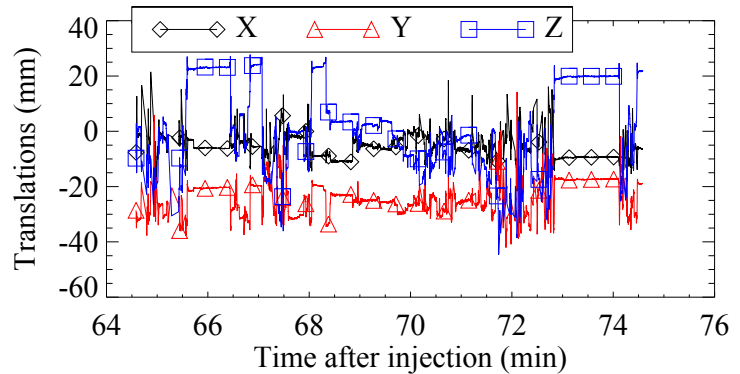

(b)

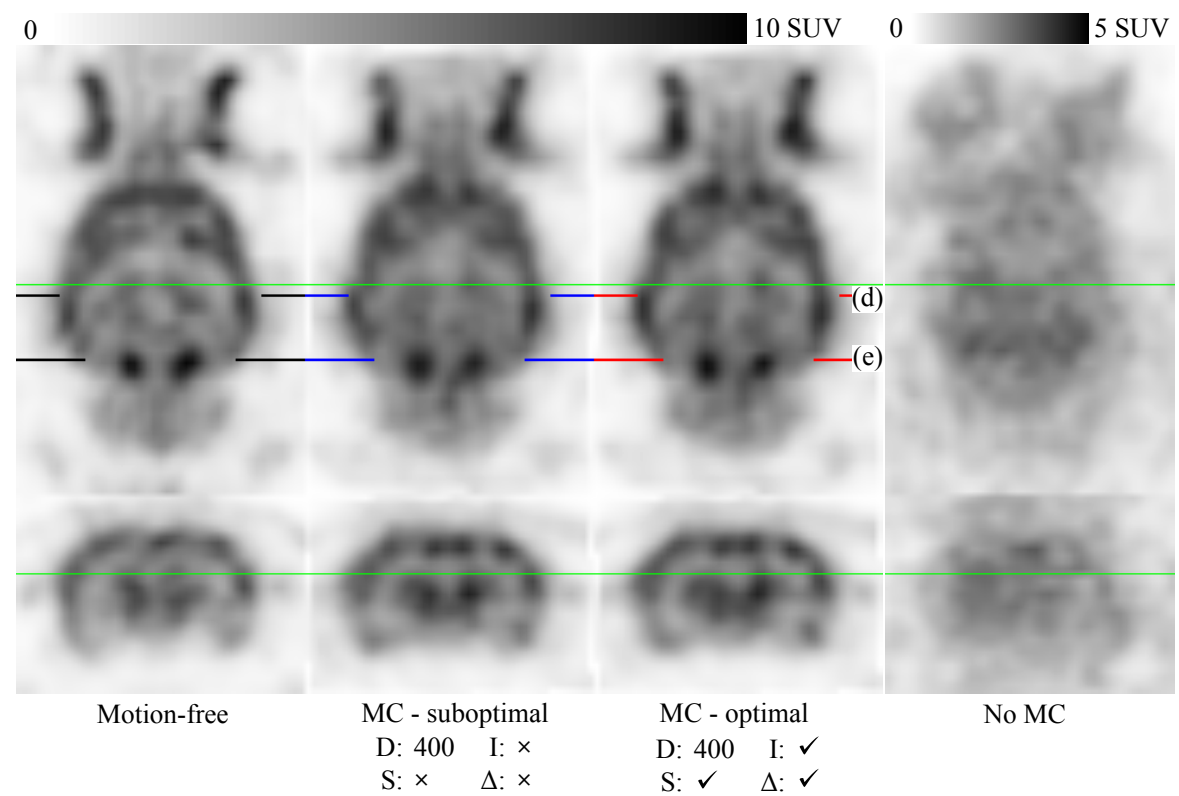

(c)

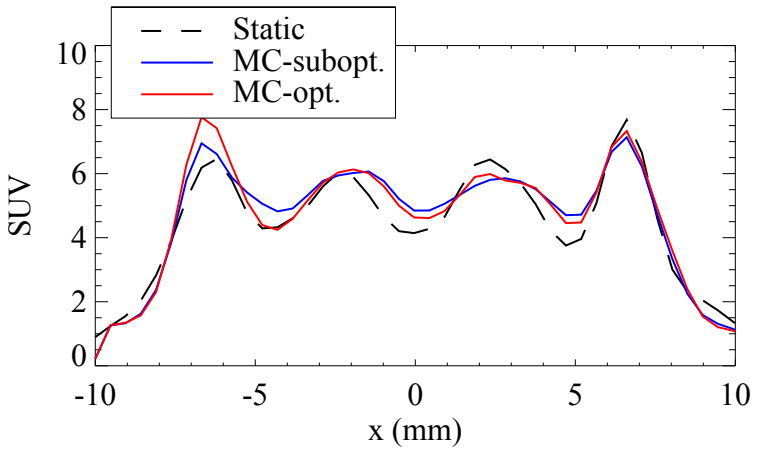

(d)

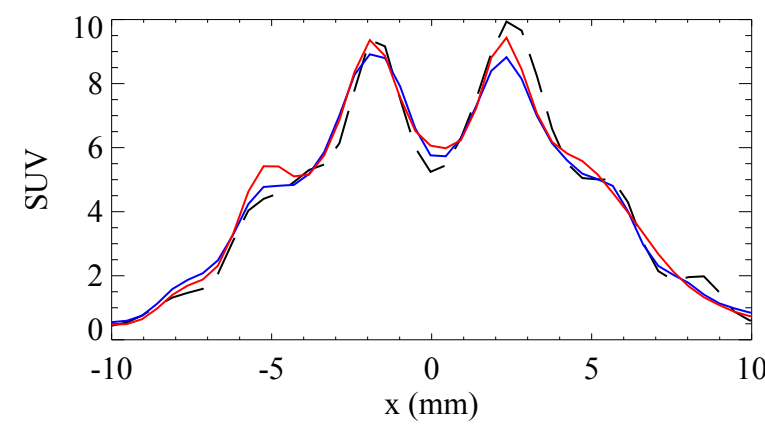

(e)

Figure 10. (a) and (b) The 6 DOF of the recorded motion in PET coordinates. (c) Reconstructed rat brain images showing a coronal (top) and transverse (bottom) slice. Results are shown for the motion-free case (first column), MC without optimisation (second column), with all optimisations (third column), and without MC (fourth column). The green lines indicate where the transverse and coronal slices are located, and the black, blue and red lines indicate where the profiles in (d) and (e) are taken. 
have been considered. The most important optimisation parameter is the pose smoothing, which, when applied alone, produces a large improvement in reconstructed image quality. Another important factor is the separation between the MT and the marker. We have shown that bringing the MT closer than the focal distance of $700 \mathrm{~mm}$ to the microPET (to between $300 \mathrm{~mm}$ and $400 \mathrm{~mm}$ ) the motion of the marker can be more accurately measured. Although the marker becomes increasingly out of focus away from the focal distance, evidently this blurring has less of an impact than the resultant magnification of the marker on accurately determining the marker pose. Our other optimisation factors - optimal delay time and pose interpolation - further improve the reconstruction, but to a lesser degree than the former two.

The synchronisation technique we describe has consistently allowed for a robust synchronisation between the MT and microPET over many experiments. When poses or gate tags are missing (due to the MT being unable to see the marker, or the microPET failing to insert a gate tag, respectively), our technique can detect and correct these gaps, in either stream, by aligning the tag and pose interval pattern throughout the scan.

The optimal delay time between the MT and the microPET was found to be on the order of a few milliseconds. While the hypothesised model did not fit the data well, the point source measurements allowed us to determine an optimal delay for the pose measurement rate we used in our studies. For a measurement rate of $30 \mathrm{~Hz}$, the optimal delay time was $-9 \mathrm{~ms}$ when no pose interpolation was used, and $14 \mathrm{~ms}$ with pose interpolation. Phantom data reconstructions were performed with various delays (not shown here) to verify, using the resolution recovery, that the determined delay was indeed the optimal. While the electronic elements involved should be similar for all microPET and MT systems, other groups should perform a similar delay optimisation experiment locally.

Using a motion-free reconstruction as the ground truth, the optimised parameters improved the resolution recovery in the MC reconstruction by approximately $27 \%$ in the phantom studies, and $14 \%$ in the rat study. During the rat experiment the rat was observed to occasionally touch the marker with its paws, which might have caused relative motion between the marker and the brain. This would in turn degrade the quality of the MC and cause blurring of both the suboptimal and the optimal reconstructions, thereby reducing the impact of the optimisations. This likely explains the discrepancy of the resolution gains between the rat and phantom experiments.

The total activity in the phantom reconstructions shown in figure 8 agree to within $1 \%$, and those in figure 9 to within $3 \%$, indicating that the MC recovers the total activity well. However, careful attention must be given to factors like synchronisation and noise to be able to resolve small structures and enable accurate quantification in such regions.

To achieve accurate quantification we will implement an attenuation correction technique where the support of the object is found from an initial MC reconstruction, and used to create an attenuation map (Angelis et al. 2013).

While this paper has focussed on hardware and software optimisations, possible marker movement relative to the brain is a potential limiting factor in awake animal 
studies and care should be taken to rigidly attach the marker. Currently however, marker-based motion tracking is the state-of-the-art for this application. A more invasive form of attaching the marker (for instance, directly to the skull) would avoid this problem, but this could have significant drawbacks (stress, complicating the procedure, etc.). Ideally, one would track the features of the head directly and avoid using an attached marker; progress towards such a technique can be found in Kyme et al. (2014), but even with such methods there is still the possibility of errors introduced by non-rigid motion of the face.

In our experience, the proposed animal training and scanning protocol has allowed us to scan many rats reliably for up to 75 minutes. In (Fulton et al. 2009) it was reported that rats in a similar set up did not have elevated stress levels. Thus we are now able to study tracer kinetics in rats without the confounding effects of anaesthesia or stress.

While this work has focused on the MicronTracker and microPET systems, many of the considered optimisations are easily translatable to other motion tracking systems and scanners used for MC. In the future we plan to investigate alternative techniques to spatially calibrate the tracker and PET systems, and to adapt and apply the presented optimisations to a clinical PET scanner.

\section{Conclusion}

Various factors have been investigated to optimise MC PET imaging of awake rats: temporal synchronisation and latency estimation between the tracker and scanner, the physical separation of the tracker and scanner, pose smoothing and interpolation, and subset selection. By optimising these factors, we have demonstrated improved quantification in MC imaging studies involving phantoms and live animals using the microPET small animal scanner and MicronTracker optical tracking system. The improvements we describe are easily translatable to other MC setups involving different scanners and motion tracking systems, both for animal and human PET imaging.

\section{Acknowledgements}

This work is supported by the IMIR project of KU Leuven and the MIRIAD Strategic Basic Research (SBO) project of the Innovatie door Wetenschap en Technologie (IWT).

\section{References}

Alstrup, A. K. O. and D. F. Smith (2013). "Anaesthesia for positron emission tomography scanning of animal brains." In: Lab. Anim. 47.1, pp. 12-18.

Angelis, G., M. Bickell, A. Kyme, W. Ryder, L. Zhou, J. Nuyts, and S. Meikle (2013). "Calculated attenuation correction for awake small animal brain PET studies". In: IEEE Nucl. Sci. Symp. Conf. Rec. 
Bickell, M. G., B. De Laat, R. Fulton, G. Bormans, and J. Nuyts (2015). "The Effect of Anaesthesia on 18F-FDG Uptake in the Rat Brain: A Fully Conscious Dynamic Study using Motion Correction". In: IEEE Nucl. Sci. Symp. Conf. Rec.

Bloomfield, P. M., T. J. Spinks, J. Reed, L. Schnorr, A. M. Westrip, L. Livieratos, R. Fulton, and T. Jones (2003). "The design and implementation of a motion correction scheme for neurological PET." In: Phys. Med. Biol. 48.8, pp. 959-978.

Bühler, P., U. Just, E. Will, J. Kotzerke, and J. V. D. Hoff (2004). "An Accurate Method for Correction of Head Movement in PET". In: IEEE Trans. Med. Imaging 23.9, pp. $1176-1185$.

Fulton, R., I. Nickel, L. Tellmann, S. Meikle, U. Pietrzyk, and H. Herzog (2003). "Eventby-Event Motion Compensation in 3D PET". In: IEEE Nucl. Sci. Symp. Conf. Rec. 2003 5, pp. 3286-3289.

Fulton, R., S. Meikle, A. Z. Kyme, V. Zhou, K. Popovic, M. Kassiou, and M. Akhtar (2009). "Motion-Corrected MicroPET Brain Imaging of Conscious Rats". In: World Mol. Imaging Conf. Montr. 12, s304-s305.

Fulton, R., S. Meikle, and S. Eberl (2002). "Correction for head movements in positron emission tomography using an optical motion-tracking system". In: IEEE Trans. Nucl. Sci. 49.1, pp. 116-123.

Horn, B. K. P. (1987). "Closed-Form Solution of Absolute Orientation Using Orthonormal Matrices". In: J. Opt. Soc. Am. A 4, p. 629.

Hosoi, R., A. Matsumura, S. Mizokawa, M. Tanaka, F. Nakamura, K. Kobayashi, Y. Watanabe, and O. Inoue (2005). "MicroPET detection of enhanced 18F-FDG utilization by PKA inhibitor in awake rat brain." In: Brain Res. 1039.1-2, pp. 199202.

Hudson, H. M. and R. S. Larkin (1994). "Accelerated image reconstruction using ordered subsets of projection data". In: IEEE Trans. Med. Imaging 13.4, pp. 601-609.

Kim, J.-H., J Nuyts, A Kyme, Z Kuncic, and R Fulton (2015). "A rigid motion correction method for helical computed tomography (CT)". In: Phys. Med. Biol. 60, pp. 20472073.

Kyme, A., J. Maclaren, S. Meikle, C. Baldock, and R. Fulton (2011a). "The effect of time domain pose filtering on accuracy of small marker based motion correction in awake animal PET". In: IEEE Nucl. Sci. Symp. Conf. Rec. 3. IEEE, pp. 2290-2294.

Kyme, A., S. Se, S. Meikle, G. Angelis, W. Ryder, K. Popovic, D. Yatigammana, and R. Fulton (2014). "Markerless motion tracking of awake animals in positron emission tomography". In: IEEE Trans. Med. Imaging 33.11, pp. 2180-2190.

Kyme, A. Z., V. W. Zhou, S. R. Meikle, and R. R. Fulton (2008). "Real-time 3D motion tracking for small animal brain PET". In: Phys. Med. Biol. 53.10, pp. 2651-2666.

Kyme, A. Z., V. W. Zhou, S. R. Meikle, C. Baldock, and R. R. Fulton (2011b). "Optimised motion tracking for positron emission tomography studies of brain function in awake rats". In: PLoS One 6.7, e21727.

Noonan, P. and J Howard (2012). "Realtime markerless rigid body head motion tracking using the Microsoft Kinect". In: IEEE Nucl. Sci. Symp. Conf. Rec. Pp. 2241-2246. 
Olesen, O. V., J. M. Sullivan, T. Mulnix, R. R. Paulsen, L. Højgaard, B. Roed, R. E. Carson, E. D. Morris, and R. Larsen (2013). "List-mode PET motion correction using markerless head tracking: proof-of-concept with scans of human subject." In: IEEE Trans. Med. Imaging 32.2, pp. 200-9.

Parra, L and H. H. Barrett (1998). "List-mode likelihood: EM algorithm and image quality estimation demonstrated on 2-D PET". In: IEEE Trans. Med. Imaging 17.2 , pp. 228-35.

Rahmim, A., P. Bloomfield, S. Houle, M. Lenox, C. Michel, K. R. Buckley, T. J. Ruth, and V. Sossi (2004). "Motion Compensation in Histogram-Mode and List-Mode EM Reconstructions: Beyond the Event-Driven Approach". In: IEEE Trans. Nucl. Sci. 51.5, pp. 2588-2596.

Reader, A., P. Julyan, H. Williams, D. Hastings, and J. Zweit (2003). "EM Algorithm System Modeling by Image-Space Techniques for PET Reconstruction". In: IEEE Trans. Nucl. Sci. 50.5, pp. 1392-1397.

Reader, A. J., K. Erlandsson, M. A. Flower, and R. J. Ott (1998). "Fast accurate iterative reconstruction for low-statistics positron volume imaging". In: Phys. Med. Biol. 43.4, pp. 835-46.

Schulz, D., S. Southekal, S. S. Junnarkar, J.-F. Pratte, M. L. Purschke, S. P. Stoll, B. Ravindranath, S. H. Maramraju, S. Krishnamoorthy, F. A. Henn, P. O'Connor, C. L. Woody, D. J. Schlyer, and P. Vaska (2011). "Simultaneous assessment of rodent behavior and neurochemistry using a miniature positron emission tomograph". In: Nat. Methods 8.4, pp. 347-352.

Shepp, L. A. and Y Vardi (1982). "Maximum likelihood reconstruction for emission tomography". In: IEEE Trans. Med. Imaging 1.2, pp. 113-22.

Stavdahl, O., A. K. Bondhus, K. Y. Pettersen, and K. E. Malvig (2005). "Optimal statistical operators for 3-dimensional rotational data: geometric interpretations and application to prosthesis kinematics". In: Robotica 23.3, pp. 283-292.

Tai, Y.-C., A. Ruangma, D. Rowland, S. Siegel, D. F. Newport, P. L. Chow, and R. Laforest (2005). "Performance evaluation of the microPET Focus: a thirdgeneration microPET scanner dedicated to animal imaging". In: J. Nucl. Med. 46.3, pp. $455-63$.

Toyama, H., M. Ichise, J.-S. Liow, D. C. Vines, N. M. Seneca, K. J. Modell, J. Seidel, M. V. Green, and R. B. Innis (2004). "Evaluation of anesthesia effects on [18F]FDG uptake in mouse brain and heart using small animal PET." In: Nucl. Med. Biol. 31.2, pp. 251-256.

Vaska, P., C. L. Woody, D. J. Schlyer, S. Shokouhi, S. P. Stoll, J. F. Pratte, P. O'Connor, S. S. Junnarkar, S. Rescia, B. Yu, M. Porschke, A. Kandasamy, A. Villanueva, A. Kriplani, V. Radeka, N. Volkow, R. Lecomte, and R. Fontaine (2004). "RatCAP: Miniaturized head-mounted PET for conscious rodent brain imaging". In: IEEE Trans. Nucl. Sci. 51.5 II, pp. 2718-2722.

Weisenberger, A. G., S. S. Gleason, J. Goddard, B. Kross, S. Majewski, S. R. Meikle, M. J. Paulus, M. Pomper, V. Popov, M. F. Smith, B. L. Welch, and R. Wojcik (2005). 
"A restraint-free small animal SPECT imaging system with motion tracking". In: IEEE Trans. Nucl. Sci. 52.3 I, pp. 638-644. 